\title{
COORDINATED VOLTAGE CONTROL OF DISTRIBUTION NETWORKS HOSTING DISPERSED GENERATION
}

\author{
Thierry VAN CUTSEM \\ University of Liège and FNRS - Belgium \\ t.vancutsem@ulg.ac.be
}

\author{
Gustavo VALVERDE \\ University of Liège - Belgium \\ gvalverde@ieee.org
}

\begin{abstract}
This paper presents a centralized control scheme to regulate distribution network voltages in the presence of dispersed generation. The algorithm resorts to model predictive control to smoothly bring unsatisfactory voltages inside the desired range of values. Using a sensitivity model, the controller calculates optimal power output changes of distributed generators to correct the network voltages, giving priority to reactive over active power. Simulations results are presented on a 32-bus test system and three variants of the optimization problem are compared.
\end{abstract}

\section{INTRODUCTION}

In the future, distribution networks are expected to host larger amounts of dispersed renewable generation. The progressive connection of dispersed sources is expected to produce voltage problems in some areas of the network. This will be aggravated by the intermittency of renewable generation which makes it more difficult to control the network according to the operator needs. Dealing with these issues will require some form of coordination between the Distributed Generators (DGs). Given the advances in communication technology and the progress on Smart Grids, it is realistic to envisage a centralized controller that can correct or mitigate those voltage problems [1]. Although a centralized control requires some investments in terms of communication infrastructure, it is considerably less expensive than reinforcing the network for temporary abnormal voltages. In order to control network voltages, it is assumed that DGs are able to change their reactive power outputs and, if needed, accept active power reductions. The active power reductions and, to a lower extent, the reactive power variations could be financially compensated by the Distribution System Operator (DSO).

In the available literature, the problem of voltage control has been formulated as single-step optimization problems, where network losses are minimized subject to voltages and line currents limits [2], [3]. These formulations typically use a sensitivity-based model. In practice, however, the sensitivity matrices can be inaccurate due to the proximity of loads whose response to voltage is not well known. In addition, a single-step optimization does not provide the smooth transition that will bring the system from its current to the targeted state. In addition, the controller should deal with measurement noise.

The aim of this paper is to outline a centralized controller inspired of Model Predictive Control (MPC) that optimally adjusts the output of DGs (active power $\boldsymbol{P}_{g}$ and reactive power $\boldsymbol{Q}_{g}$ ) to maintain the monitored voltages within a pre-defined target range of values, determined from security or economic considerations.

This paper reports on an extension of the authors' previous work [4]: to improve the controller response, an additional term is used in the objective function, which penalizes the deviations of the voltages with respect to the mid-point of the above-mentioned target range.

\section{MULTI-STEP OPTIMISATION ALGORITHM}

The proposed control uses a sensitivity model to predict the behavior of the system over a future interval of $N_{p}$ discrete steps, in response to an optimal sequence of discrete control actions at $N_{c}$ future steps $\left(N_{c} \leq N_{p}\right)$.

At discrete time $k$, the controller determines the optimal changes of the control variables $\boldsymbol{u}$, at instants $k, k+1$, ..., $k+N_{c}-1$ with the objective of progressively bringing the monitored voltages inside the desired interval. According to the very principle of MPC, only the first control action $\Delta \boldsymbol{u}(k)$ of the sequence is applied at time $k$, with:

$$
\Delta \boldsymbol{u}(k)=\boldsymbol{u}(k)-\boldsymbol{u}(k-1)=\left[\Delta \boldsymbol{P}_{g}(k)^{T}, \Delta \boldsymbol{Q}_{g}(k)^{T}\right]^{T}
$$

Note incidentally that (1) may also include the voltage set-point of the load tap changer controlling the transformer which feeds the network, as detailed in [4]. At the next time step, based on the newly available measurements, the whole control sequence is recomputed and, again, only the first step is applied [5]. This receding-horizon scheme allows compensating for model inaccuracies and measurement noise.

At time $k$, the following quadratic objective is considered:

$$
\min \sum_{i=0}^{N_{c}-1}\|\Delta \boldsymbol{u}(k+i)\|_{\boldsymbol{R}}^{2}+\sum_{i=1}^{N_{p}}\|\boldsymbol{r}-\boldsymbol{V}(k+i \mid k)\|_{\boldsymbol{T}}^{2}+\|\boldsymbol{\varepsilon}\|_{\boldsymbol{S}}^{2}
$$

where the first term aims at minimizing the total control effort, and the second penalizes the deviations of the predicted voltages $\boldsymbol{V}(k+i \mid k)$ with respect to the reference $\boldsymbol{r}$. The notation $\boldsymbol{V}(k+i \mid k)$ stands for the predicted voltage at instant $k+i$ given the measurement at time $k . \boldsymbol{R}$ and $\boldsymbol{T}$ are diagonal weighting matrices.

The slack variables $\varepsilon=\left[\varepsilon_{1}, \varepsilon_{2}\right]^{T}$ are used to relax the operation constraints in case of infeasible solutions, as discussed in [4]. These variables are heavily penalized in 
(2), using the weighting matrix $\boldsymbol{S}$, to keep them at zero whenever possible. The constraints are:

For $i=0,1, \ldots, N_{c}-1$ :

$$
\begin{gathered}
\boldsymbol{u}^{\min } \leq \boldsymbol{u}(k+i) \leq \boldsymbol{u}^{\max } \\
\Delta \boldsymbol{u}^{\min } \leq \Delta \boldsymbol{u}(k+i) \leq \Delta \boldsymbol{u}^{\max }
\end{gathered}
$$

For $i=1, \ldots, N_{p}$ :

$$
\begin{gathered}
-\varepsilon_{1} \mathbf{1}+\boldsymbol{V}^{\min }(k+i) \leq \boldsymbol{V}(k+i \mid k) \leq \boldsymbol{V}^{\max }(k+i)+\varepsilon_{2} \mathbf{1} \\
\boldsymbol{V}(k+i \mid k)=\boldsymbol{V}(k+i-1 \mid k)+\frac{\partial \boldsymbol{V}}{\partial \boldsymbol{u}} \Delta \boldsymbol{u}(k+i-1)
\end{gathered}
$$

The control limits $\boldsymbol{u}^{\min }$ and $\boldsymbol{u}^{\max }$ are set according to the capabilities of the DG, while $\Delta \boldsymbol{u}^{\min }$ and $\Delta \boldsymbol{u}^{\max }$ relate to the maximum rate of change allowed for the controls.

The limits $\boldsymbol{V}^{\min }(k+i)$ and $\boldsymbol{V}^{\max }(k+i)$ are the voltage limits at the $i$-th prediction step, while $\mathbf{1}$ denotes a unitary vector. A progressive tightening in time of these voltage limits is considered, so that their values at time $k+N_{p}$ coincide with the specified voltage bounds [4].

The predicted voltages in $(2 \mathrm{~d})$ are approximated by computing their sensitivity with respect to the control variables $\partial \boldsymbol{V} / \partial \boldsymbol{u}$. This sensitivity matrix can be obtained from the inverse of the Jacobian matrix extracted from an offline power flow calculation [4].

\section{SIMULATION RESULTS}

A 32-bus, 20-kV distribution network with four DG units has been used to test the proposed control algorithm. The network topology and line parameters were taken from [6]. The one-line diagram is shown in Figure 1.

The distribution system is connected to the HV external grid through a HV/MV transformer with fixed tap position, and it serves 12 loads modelled as constant current for active power and constant impedance for reactive power, plus three equivalent induction motors.

The controller was implemented in RAMSES, a detailed time simulation program developed at the Univ. of Liège. The controller is assumed to send control changes $\Delta \boldsymbol{u}(k)$ every $10 \mathrm{~s}$; those variations are added to the $\boldsymbol{P}_{g}$ and/or $\boldsymbol{Q}_{g}$ set-points of the various DG dynamic models.

The control and prediction horizons were fixed at $N_{c}=N_{p}=3$. For demonstration purposes, we provide simulation results for the test system initially operating at undesirable voltages. The aim of the controller is to bring all voltages within the range [1.005 1.015] p.u.

The four DGs are assumed to be synchronous machines, which have slower responses than power-electronics devices and, hence, leads to more stringent tests. The active power output of each machine is regulated by a Proportional and Integral (PI) controller to meet the demands of the centralized controller under emergency conditions, i.e., only when a monitored bus voltage is found outside [0.940 1.060] p.u. Please refer to [4] for details.

Each generator is also controlled by an Automatic Voltage Regulator (AVR) with an inner control loop to regulate the terminal voltage in response to fast changes, and an outer PI control loop to adjust the reactive power to the requested set-point.

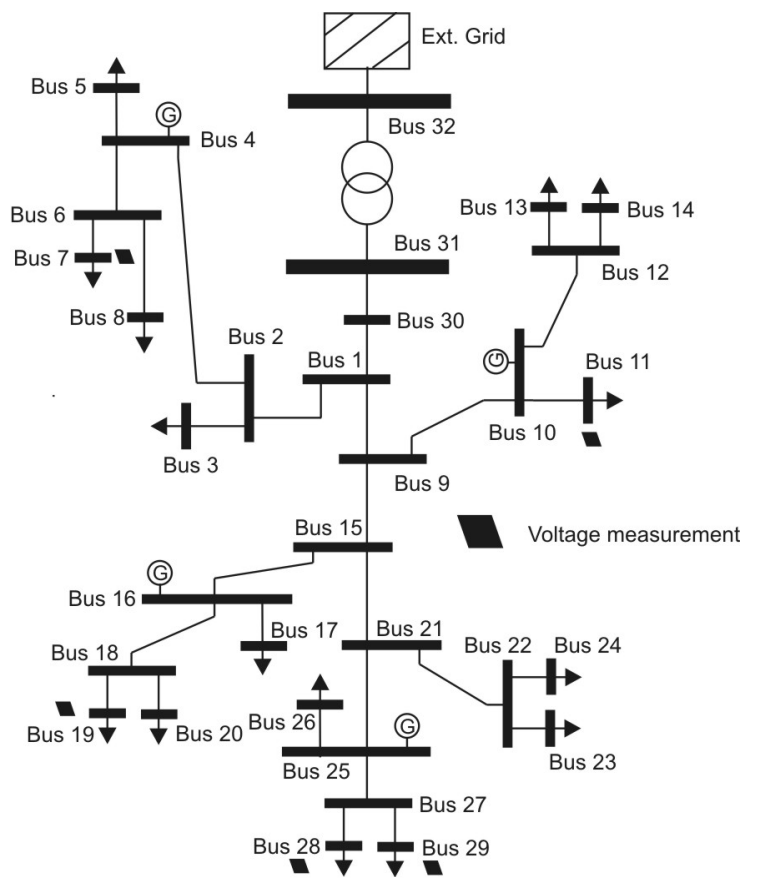

Figure 1: One-line diagram of the test system

It is assumed that measuring devices collect and transmit to the controller the following data: the active power, reactive power and voltage magnitude at the terminals of the four DGs, and the voltages at the load buses 7, 11, 19, 28 and 29. The location of these devices has not been optimized. However, by distributing them all over the network, it is reasonable to expect that the voltages of non-monitored buses will be close to the voltages of the neighbouring measured buses.

The noisy measurements are collected some time after the control actions are applied. This is to wait for the system response and to avoid making decisions based on measurements taken during transients [4]. These noisy measurements were simulated by adding white Gaussian noise restricted to $\pm 1 \%$ for $\boldsymbol{V}$ measurements and $\pm 1 \%$ of the respective DG maximum power output for $\boldsymbol{P}_{g}$ and $\boldsymbol{Q}_{g}$ measurements.

The actual voltage dependency of loads is uncertain in practice. Therefore, the sensitivity of bus voltages with respect to power injections was calculated from a power flow calculation assuming constant power loads.

\section{Case 1}

In the first test case, the controller does not penalize the deviations of monitored voltages with respect to the reference $\boldsymbol{r}$, i.e. $\boldsymbol{T}=\mathbf{0}$ in (2). Additionally, in the first term of (2), all changes of reactive power outputs have the same weight, equal to one.

Figure 2 presents the voltage evolutions at four monitored load buses. 


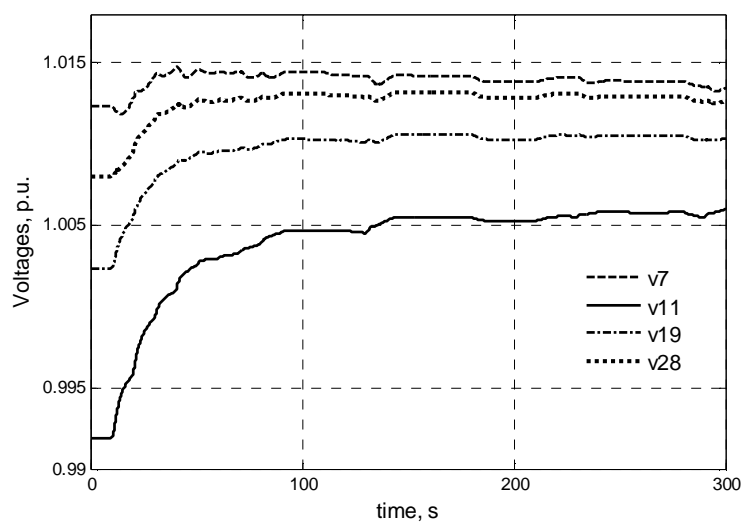

Figure 2: Voltage evolutions for Case 1

The controller detects that some voltages are outside the desired range at around $t=5 \mathrm{~s}$. By solving (2), the controller applies the optimum $\Delta \boldsymbol{Q}_{g}$ to correct all monitored bus voltages at $t=10 \mathrm{~s}$ and every $10 \mathrm{~s}$ thereafter. All voltages enter the desired range of values at around $t=150 \mathrm{~s}$. Note that further corrections after this time are triggered by the noisy measurements received by the controllers, but they are very small.

For the time instants when the measured voltages were all inside the desirable range of values, the controller did not request any $\Delta \boldsymbol{Q}_{g}$.

Figure 3 presents the corresponding evolution of the reactive power outputs of $\mathrm{DG}$ units. Here, the generator at bus 4 is requested to increase its reactive power output to correct v11 and v19. At the same time, the remaining generators are requested to reduce their reactive power outputs to avoid over-voltages at the other monitored buses.

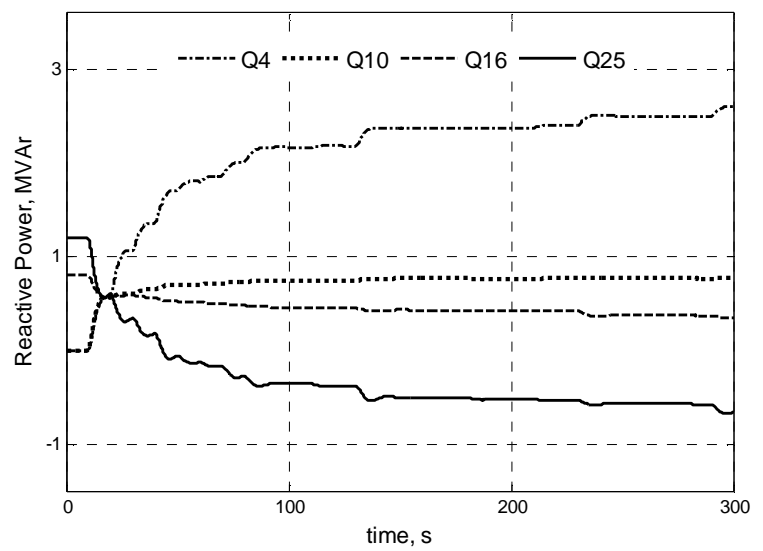

Figure 3: DG reactive power outputs for Case 1

The DGs active powers are left unchanged because the controller can use them only in the above mentioned emergency conditions.

\section{Case 2}

Starting from the same operating conditions as in Case 1, in this test the deviations of the monitored voltages with respect to the reference (1.01 p.u.) are penalized, through the second term in the objective (2), with all weights set equal to one.

Figure 4 presents the voltage evolution at the monitored load buses. Due to the penalization of voltage deviations, the controller not only brings all voltages within the requested limits but also brings them closer to the 1.01 p.u. reference.

From Figure 4, it is seen that by penalizing the voltage deviations, the controller takes approximately $65 \mathrm{~s}$ to bring all voltages within the desired limits. This is significantly less time than in Case 1. This faster response, however, is achieved at the expense of a temporary violation of v7 (> 1.015 p.u.) and larger DG reactive power changes, as shown in Figure 5.

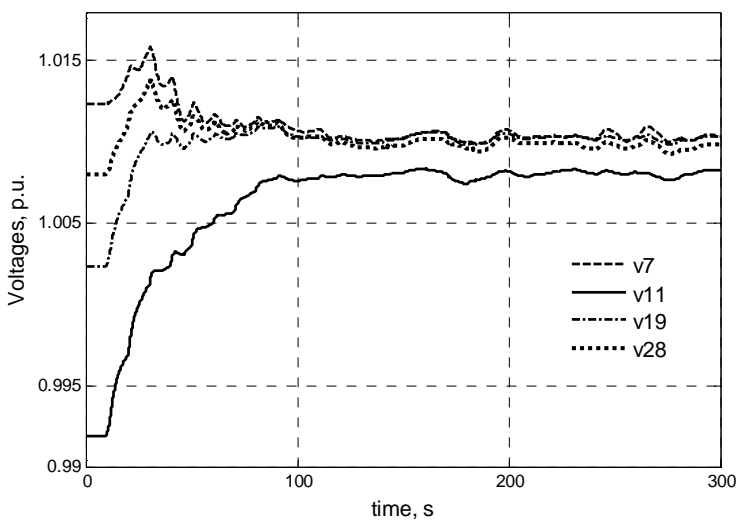

Figure 4: Voltage evolutions for case 2

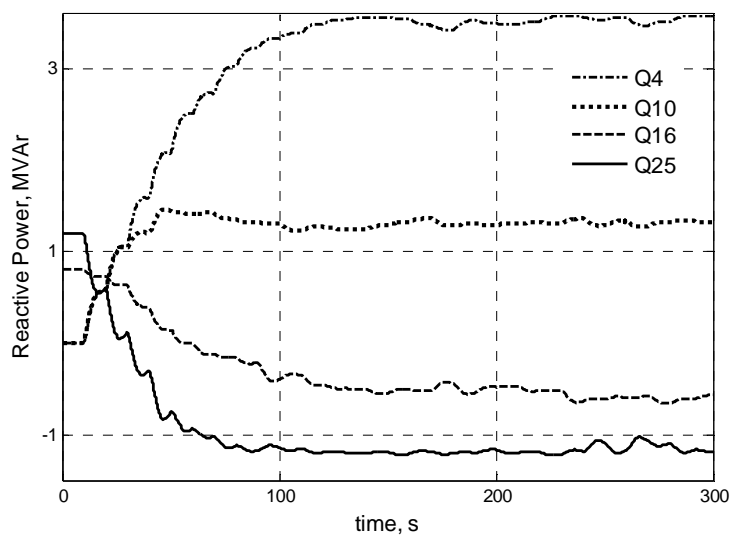

Figure 5: DG reactive power output for Case 2

Furthermore, as voltage deviations are penalized, the controller requests changes of the DG reactive power outputs even when the controlled voltages are already within the desired limits. This explains the power output fluctuations for $t>70 \mathrm{~s}$.

Each of the previous cases has its own benefits and drawbacks. For example, with the implementation of Case 1 the corrections are very smooth and saturation of the control variables is avoided. For faster control responses, the implementation of Case 2 should be considered. However, the latter tends to saturate the 
control variables and, regardless of voltage limits, it keeps correcting the voltage deviations with respect to the reference $\boldsymbol{r}$. The main problem is that due to model inaccuracies, system uncertainty and measurement noise, it is almost impossible for the controller to reach these voltage references, as confirmed by the results of Figure 4. A compromise between these two cases seems to be appropriate, as considered in the next case.

\section{Case 3}

This case combines the previous two approaches as follows:

- the controller penalizes voltage deviations from 1.01 p.u. if the monitored voltages are outside the targeted [1.005 1.015] p.u. range;

- once all voltages are within these limits, the second term in (2) is dropped (i.e. $\boldsymbol{T}=\mathbf{0}$ ) to avoid unnecessary $\Delta \boldsymbol{Q}_{g}$ requests.

Figure 6 presents the bus voltage evolutions for Case 3 . Note that due to the penalization of voltage deviations, the controller has the same time response as in Case 2. However, when the voltages are all inside the desired range of values, the controller does not request further changes of reactive power outputs, as in Case 1.

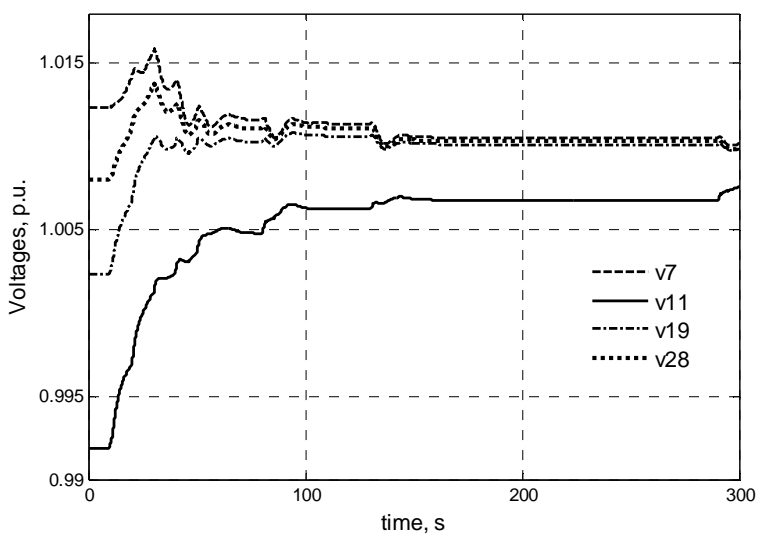

Figure 6: Voltage evolutions for Case 3

Figure 7 presents the reactive power outputs of the DG units. Initially, the controller requests large $\Delta \boldsymbol{Q}_{g}$ changes to minimize the second term in (2) as quickly as possible (within the limits imposed by (2b) though). When the controller detects that all voltages are within [1.005 1.015] p.u., it stops penalizing the voltage deviations and the DGs are not requested to change their reactive power outputs until the voltages go outside the limits again. This good control strategy avoids frequent voltage corrections.

Note that the reactive power changes observed at $t=130 \mathrm{~s}$ and $t=290 \mathrm{~s}$ were triggered by the noisy measured voltages that led to adjust the DG reactive power set-points.

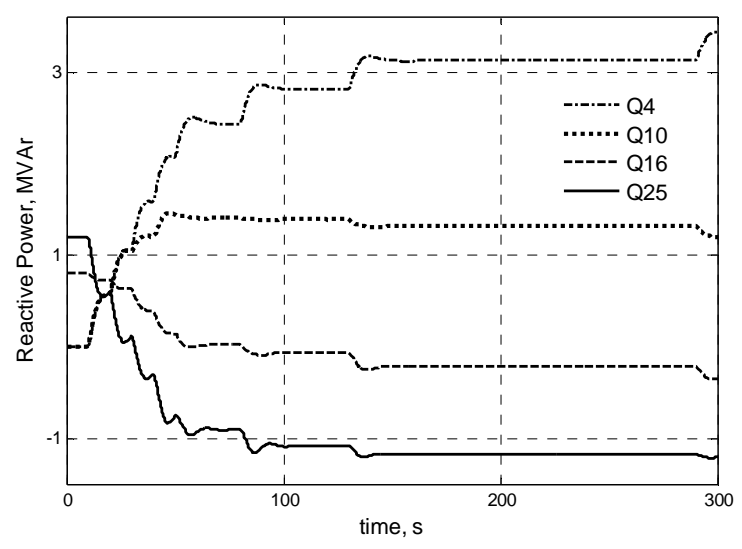

Figure 7: DG reactive power output for Case 3

\section{CONCLUSION}

This paper presented a centralized control scheme based on MPC to correct distribution network voltages. It was tested on a detailed dynamic model of DGs. The corrections are made by computing and applying a sequence of optimal control changes of the DG power outputs.

The simulations show that the controller response is faster when penalizing the deviations between monitored and reference voltages.

Due to model inaccuracies and noisy measurements, it is impractical and maybe impossible to reach these reference voltages. However, if the voltage deviations are penalized only when the voltages are outside the requested range of values, the algorithm combines fast response and less frequent DG output changes.

\section{REFERENCES}

[1] O. Richardot, A. Viciu, Y. Bésanger, N. Hadjsaïd, C. Kieny, 2006, "Coordinated voltage control in distribution networks using distributed generation" PES Transmission and Distribution Conference and Exhibition, IEEE, 11961201.

[2] A. Borghetti, M. Bosetti, S. Grillo, S. Massucco, C.A. Nucci, M. Paolone, F. Silvestro, 2010, "Short-term scheduling and control of active distribution systems with high penetration of renewable resources," IEEE Systems Journal, vol. 4, 313-322.

[3] Q. Zhou, J.W. Bialek, 2007, "Generation curtailment to manage voltage constraints in distribution networks" IET Generation Transmission and Distribution, vol. 1, 492498.

[4] G. Valverde, T. Van Cutsem, 2013, "Model predictive control of voltages in active distribution networks," submitted to IEEE Transactions on Smart Grids.

[5] J.M. Maciejowski, 2002, Predictive Control With Constraints, Prentice-Hall, New Jersey, United States.

[6] E. Kagi-Kolisnychenko, 2009, "Distribution management system including dispersed generation and storage in a liberalized market environment" Ph.D. dissertation, Ecole Polytechnique Federale de Lausanne, Switzerland. 